\title{
A Robust Method to Detect Coverage Holes and Network Re-Establishment for Nb-Iot Enabled Networks
}

\author{
Anbumani M, Thenmalar S
}

\begin{abstract}
Sensing coverage with respect to WSN (Wireless Sensor Network) research has gained immense consideration. Implementation of WSNs in the domain of IoT (Internet of Things) ensures consideration of IoT features when thinking of sensing coverage. Both IoT and WSN are with respect to coverage hole metrics that includes network re-establishment, link establishing, throughput etc. Implementing sensor nodes may result in the issue of coverage holes if there is improper deployment of nodes. A coverage hole can exist in any region that is being monitored at any instance due to various purposes. As a result detecting coverage hole stand as a prime concern for gaining absolute coverage. Detecting coverage hole pose a big question for gaining high coverage in the Wireless Sensor Networks. The problem of coverage and connectivity particularly in coverage deployment strategy is analyzed for the weaker nodes and in case network design fails. The proposed algorithm referred to as 'Store and Forward technique' aids in identifying the coverage holes and network re-establishing in WSNs and IoT. Hence with the help of Store and Forward technique, network re-establishing and network stability can be performed in case of a coverage hole issue. The proposed technique is mostly employed in networks having irregular connectivity, specifically within environments where mobility is high. It's also beneficial in conditions with long transmission and variable delays, maximum error rates, or cases where there is no direct, end-to-end link. The experiment output represents that the proposed healing method magnifies coverage rate with only a few additional sensors in contrast to the related methods.
\end{abstract}

Keywords-wireless sensor network (wsn), internet of things (iot), quality of service (qos), coverage hole detection, store and forward technique, network reestablishment, network reconfiguration

\section{INTRODUCTION}

Wireless sensor networks (WSNs)have their application in varied domains like for instance battlefield-surveillance [1], target-tracking [2], environment-monitoring [3], and health care [4]. All the above mentioned domain are bound to be target-critical and it's essential that the area under supervision must be thoroughly covered so that there is

Revised Manuscript Received on July 22, 2019

M. Anbumani, M.TECH , Department of Computer Science and Engineering, SRM Institute of Science and Technology, Chennai ,India (Email: anbudino09@gmail.com)

S. Thenmalar, Assistant Professor, Department of Computer Science and Engineering, SRM Institute of Science and Technology ,Chennai ,India accurate identification of the events happening in the targeted area. Though such applications frequently confront the issue of coverage holes which brings down the performance of the WSNs, by minimizing the connectivity, distorting the WSNs load and worsening the burden of holeborder-nodes while data transmitting. Usually the occurrence of coverage holes symbolizes explosion of critical events like earthquake, forest fire etc. Regrettably, the appearance of coverage holes is uncontrollable because of reasons like: nodes failure, non-uniformity of deployment, and terrain hurdles, and keep on magnifying in size and number with time. Hence there is an urgent need for lessening the growth of coverage holes. Irrespective of any approach being implemented, identification of coverage holes is of prime concern rather than optimizing routing protocols or minimizing energy utilization. Prevailing Narrow Band-IOT on-board sensors having intelligent multimodal sensing component thoroughly employs the spatial correlation and mutual cooperation. Occurrence of Coverage hole can be because of death of a standard node or energy hole. The region around coverage hole cannot be sensed. Hence, there is a necessity of employing intelligent and effective energy for delaying the lifetime of network as well as preventing eruption of network problems and coverage holes. Concerning this, network efficient routing stands to be promising than rest of the techniques of energy conservation for conservation of nodes' sensors. Coverage holes are also created because of deploying the nodes randomly, that is when the nodes are not distributed evenly within the network area. Resultant, some of the network region gets more populated whereas leaving the remaining area least populated making it highly prone to coverage holes. A powerful approach is being proposed for the identification of coverage hole and reestablishment of network for the narrow band-IoT enabled networks. At the beginning, depth based routing is examined that assists in detecting those spots which utilizes immense energy. Thereafter a technique is recommended for balancing the energy utilization of sensor nodes. The proposed technique of 'store and forward' detects unnecessary coverage areas and shifts the nodes from redundant coverage areas to fill up the hole area. The study focuses on establishing network stability and reconfiguration

that's headed towards the planning stage.

The researches aim to reduce the construction cost keeping the supply quality aside. The first identified work that tries to resolve the issue of distribution system reconfiguration concerning loss reduction. To achieve and improvise network stability using the proposed

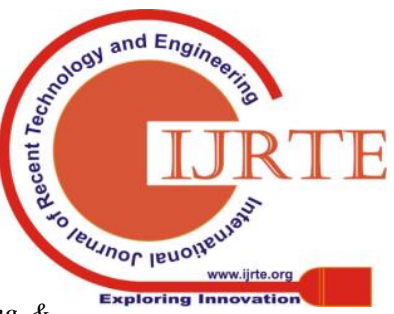




\section{A Robust Method to Detect Coverage Holes and Network Re-Establishment for Nb-Iot Enabled Networks}

method no extra cost is required for routers installation, switches and network tools within the communication systems.

Following is the classification of the journal: Section 2 presents work of previous author. Section 3 put forward the proposed ML (machine learning) techniques and various stages outlook. Section 4, presents experimental outcome and lastly, Section 5 give the conclusion concludes the paper and proposes future research work.

\section{RELATED WORK}

Li-Hui Zhao et.al emphasizes on detecting the coverage holes and boundary nodes, which being extremely essential so as to avoid enlargement of coverage holes and making sure that data is well transmitted. The issue of coverage holes is being highlighted and concerning the same two novel algorithms are proposed which can detect these coverage holes. First algorithm being the DSCS (Distributed Sector Cover Scanning), which helps in detecting the nodes near hole borders and external boundary of wireless sensor networks. The second algorithm being DW (Directional Walk), which can identify these holes by relying on the boundary nodes that are detected using DSCS [5].

Huang et al. outspread the approach of is contour and propose a scheme that utilizes the two-hop neighbors of any given node so as to create anis contour of it. In case the isocontour is broken, the node represents the boundary node. Though the node location is not needed by the scheme, numerous boundary nodes are being marked [6].

Abdullahi B. Kunya et.al proposes the technique of BPSO (Binary Particle Swarm Optimization) that being implemented for optimal network re-configuration concerning the primary distribution network. The proposed method aims to reduce overall active power loss and voltage variation found in the network. It's dependent on multiple technical constraints, with the search space represented with a group of networks branches. IEEE 16-bus, 33-bus and 69bus test distribution networks are being adopted to ensure the methods efficiency and its actual workability on multi-scale distribution systems [7].

Ma, H. C., et.al, presents a 3D coverage and deployment strategy for examining sensing features by utilizing the cuboid structures. For obtaining the output the authors makes use of $0-1$ sphere sensing model for sensing. Initially, a nodes count is determined for achieving throughout coverage in 3D. Thereafter a 3D-grid-triangulation of the network region is made, the positions are determined for deploying sensor nodes. The authors recommend that the sensing model from 0 to 1 sphere can stretch out to probabilistic sensing model. Additionally, there can be an extension using mobile sensor nodes or towards the concept of k-coverage [8].

Ammari, H. M et.al, considers the k-coverage concept in 3dimensional WSNs via intersection of convex sets. The authors employs the geometry of reuleaux tetrahedron thereby determining the least sensor spatial density for overall k-coverage in 3 dimensional domain. In addition, on the basis of spatial density, the authors confirms that the connectivity in 3D k-covered sensor networks is higher compared to the k-sensing coverage. Alongside, computation concerning the conditional connectivity in 3 dimensional kcovered wireless sensor networks is carried out [9].
Babaie, $S$ et.al, presents a snapshot for the healing of coverage holes in 2D WSNs for mobile nodes. The size and location of coverage holes are predicted and thereafter the mobile nodes are re-localized based on the triangular oriented diagram, for healing such holes [10].

Kang, $\mathrm{Z}$ et.al, presents a working on the algorithm of coverage hole detection. This algorithm has the potential of decentralization and freedom of co-ordinates. The hole repairing algorithm is brought into practice by relying upon the geometry of the perpendicular bisectors and also by considering the proposed hole detection algorithm. The output assures and reveals full coverage [11].

Kamran Latif et.al, presents the concerns related to the techniques of depth based routing namely, DBR and EEDBR. Various procedures/processes in the protocol operation are determined which consumes immense energy. (Like, in KSP and static transmission power level). As a solution, a technique is recommended for minimizing energy consumption in KSP along with the usage of various transmission power levels. With these improvisation, network lifetime and throughput is made better. The hole repair technique is introduced for addressing the problem of coverage hole. The technique works by first searching the redundant coverage overlapping of nodes near by the coverage hole, and thereafter rectifying this hole by moving nodes from the redundant coverage area. Though, this sort of node movement is limited in overcoming the building up of new coverage hole. At last, improved performance of the work is verified with respect to the selected performance metrics via comparative simulations [12].

C. Ma et.al, recommends a technique for repair of nodes in the coverage hole using fuzzy logic. In network area, at the time of deployment, mobile nodes are being introduced. On the death of a node, the dead node is identified and it's determined as to which of the mobile node will move to fill up the hole. The protocol yields in satisfying performance in case of low death rate of nodes, but the performance decreases in case there of higher death rate of nodes as covering the holes with restrictive no: of mobile nodes becomes quite difficult. The protocol targets on hole repair particularly concerning the 2D deployment of nodes [13].

P. K. Sahoo et.al, presents a Distributed Coverage HORA (Hole Repair Algorithm) for wireless sensor networks created in mobility feature for overall nodes. During the start of network operation, the neighbor information (concerning the overlapping of sensing ranges) is recorded by all the nodes. On the death of a node, the hole gets filled by the neighbouring nodes. The node having the topmost priority is moved first which is being determined by the distributed algorithm.

HORA makes sure that there is no new coverage hole being created when a node moves to fill the coverage hole that exists. In addition, the work put forth a $2 \mathrm{D}$ mathematical model to sense the nodes disk overlapping [14].

Yunzhou Zhang et.al, discusses a new methodology for detecting coverage hole using residual energy in WSNs that are randomly deployed. The life 


\section{A Robust Method to Detect Coverage Holes and Network Re-Establishment for Nb-Iot Enabled Networks}

expectancy of working nodes is computed via residual energy, for making an adjustment amidst the cost of network repair and energy waste. The working nodes having a short life span are scanned based on a proper ratio. Thereafter, the location of coverage holes can be ascertained by computing the evaluation criteria and joint coverage probability [15].

Martins et.al, identifies the coverage holes by utilizing the approach of Rips complex and Cech complex. In case the communication radius $(>=)$ twice the sensing radius with the presence of a hole in Rips complex, then there do exist a hole in Cech complex. The holes can be triangular or nontriangular as denoted by the author. According to the proposed distributed algorithm the non-triangular holes and the area of triangular holes can possibly be identified. Once the neighbor graph is formed, the existence of Hamiltonian cycle in graph is verified by every node. In case it's not then the node resides on the hole boundary. Once the decision is made, each node transmits its status to its neighbors. Thereafter, the cycles bounding holes are detected by the algorithm [16].

Zeadally et.al proposes a hop based technique for identifying holes within WSNs. It includes three phases, first being the information collection phase in which information is exchanged by every node to create a list of $\mathrm{x}$-hop neighbors, second phase is of path construction where referring the list of x-hop neighbors, communication links between sensor nodes are detected and third being the path checking phase which analyzes the paths for inferring boundary and inner nodes. In case the communication path of x-hop neighbors of a node is broken, then it's assumed to be a boundary node. Algorithm performance is quiet good for node degree of 7 or more compared to rest of the approaches, thought it involves a large communicationoverhead in detecting $\mathrm{x}$-hop neighbors [17]. DBD(decentralized boundary detection) for the identification of sensor nodes near a hole or hurdle within sensor networks by employing the topological approach. Every node is aware of its three-hop neighbors with exchange of HELLO messages and one-hop and two-hop node information. No UDG constraint is present. Thereafter, a 2-hop neighbor graph is built by every node. In case a cycle is present in the graph, it signifies a hole in network. To handle holes that are not completely within 2-hop neighbor graph, a different rule based on contour structure is built. If a broken contour line is identified, it resembles the presence of a network boundary or an obstacle [18].

Pearl Antil et.al, presents discussion on various kinds of holes and importance of hole detection in WSNs. Schemes for coverage hole detection are categorized into the following three categories: the information type utilized by the algorithms, computation model, and network dynamics for improved comprehension. Thereafter a discussion is carried out emphasizing on the relative strengths and drawback of few prevailing coverage hole detection algorithms [19].

Wei Li et.al, proposes a novel algorithm by incorporating the features of empty circles for assessing if the coverage holes is present or not in sensor networks and detecting precise nodes near the holes boundaries. The proposed algorithm is split in two sections: (1) detecting coverage hole
Chu and Ssu et.al, proposes the algorithm of

and coarse boundary nodes clustering (2) refining of boundary nodes. Using theoretical proofs, the precision of the algorithm is illustrated [20].

AnjuSangwan et.al, presents various situations referring to the sensor nodes deployment or network topology taking into account various shaped coverage holes. Additionally, for healing the holes, sensor nodes containing sensing radius separate from that of initial deployed sensors is introduced. This results in the growth and advancement of heterogeneous sensor networks, a seeking concern in terms of technology development. The founding heterogeneous sensor networks due to such sensor deployment affects the whole network cost related to reduction of cost with an optimum coverage level. Using a theorem, the mathematical analysis to frame a link between the radii of former and later deployed sensor nodes can be illustrated. [21].

\section{PROPOSED WORK}

\section{A. Overview}

The 2 dominating performance metrics namely, coverage and network connectivity are responsible for the development of WSNs considering developing technologies. With the existence of a coverage hole there exists a break within the network lifetime. Hence it's highly important to attend any such coverage holes in sensor networks. Presence of these holes at any network levels may and does impact the overall performance. On the basis of intense study it's illustrated that avoidance of coverage holes can result in redundant energy consumption of the boundary nodes. Hence it's extremely mandatory to identify and heal the coverage holes for hassle-free and effective running of WSN. With the help of proposed Store and Forward technique, network reestablishment and network stability can be performed in case of a coverage hole issue. This techniques aids in deployment and re-configuring the network in case of coverage hole detection.

\section{B. Coverage Holes in WSN}

Region where the working of few sensor nodes is hampered and there is no process of data sensing and communication is referred to as a 'hole'. Holes are considered as a hindrance in the process of communication. They highly affect the networks performance. The process of detecting coverage hole involves identification of attacked, damaged or inaccessible nodes. In case a hole exists in the sensor network the data gets routed repeatedly near the boundary nodes of the hole which results in untimely energy consumption of such nodes. This in turn expands the hole-

size unnecessarily which further leads to congestion. Detection of such holes promises high life span of the network and effective QoS (quality of service). Detection of network boundary, i.e. nodes residing around the boundary of network as well as surrounding the hole is a challenging concern in the wireless sensor networks.

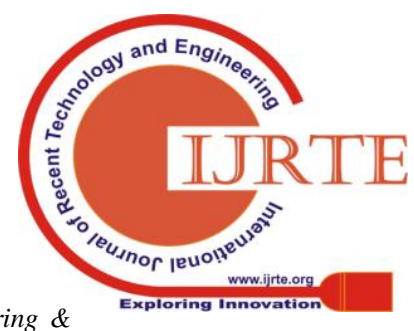
Sciences Publication 


\section{A Robust Method to Detect Coverage Holes and Network Re-Establishment for Nb-Iot Enabled Networks}

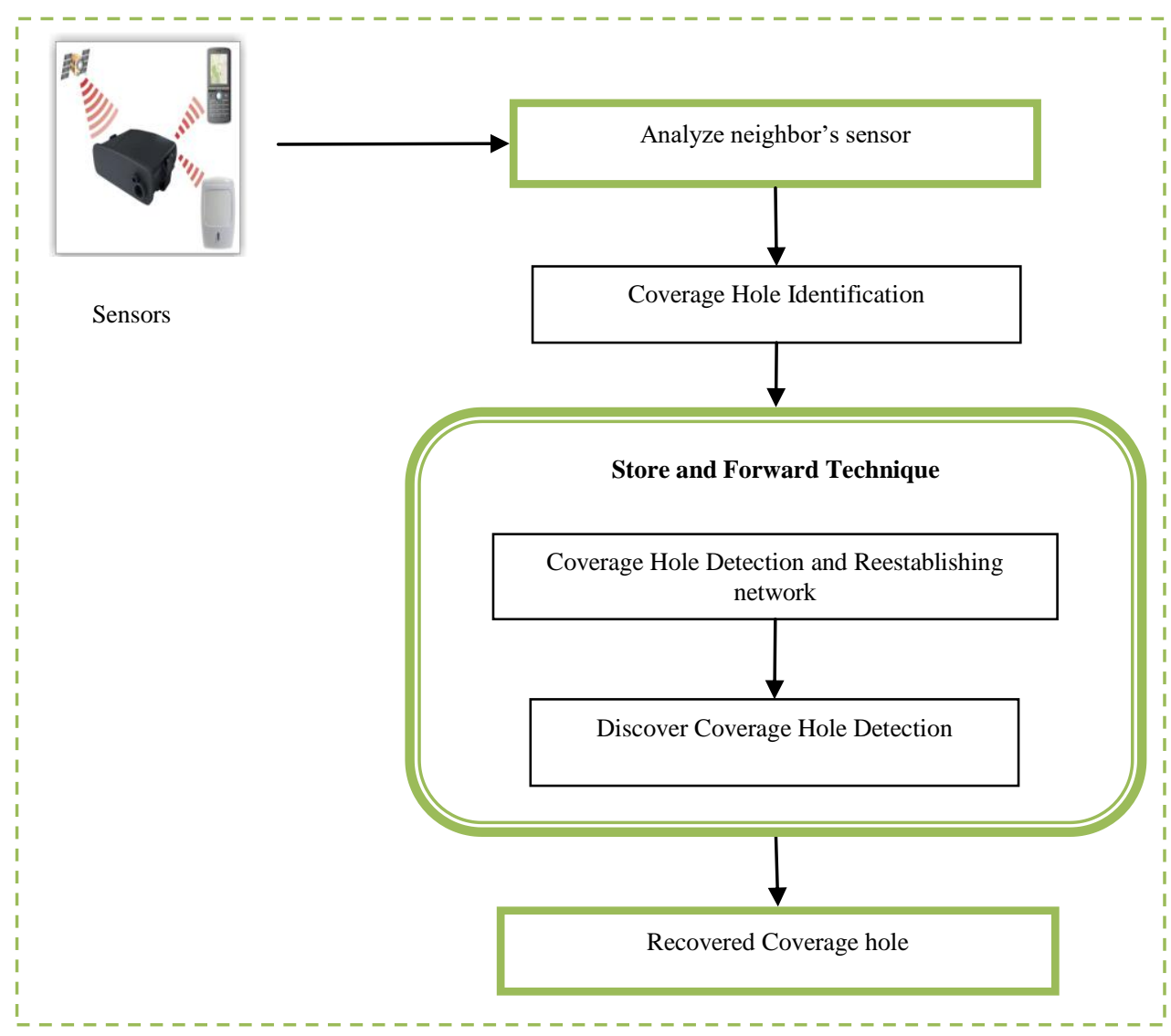

Fig 1: Proposed Architecture

\section{Node Selection}

Identifying the precise sensor nodes that closely lie around the obstacles in order to create tight sensors. Initially, few of the sensor nodes residing close to the obstacles are identified. Each of the sensor nodes links itself with its two adjacent lying sensor nodes that being nearest to the obstacle on its either side. At last, the sensor nodes make a connection with one another at a time (i.e. one by one) circling the obstacles so as to build complete sensor boundary. Relying upon the above sensor nodes, rest other sensor nodes are determined. Every single initiated nodes individually chooses two adjacent nodes as sensor nodes besides its either side (i.e. left and right). The nodes that are selected lies within initiated sensor nodes one-hop communication range and closest to the obstacle. On the basis of the connection process of every node to its either side nodes, the nodes connect to the other one at a time, forming a complete node boundary.

\section{Coverage hole detection}

When the working of few sensor nodes is hampered in a region and there is no process of data sensing and communication then there is definitely a hole present in the network. They highly affect the networks performance. Here the point area coverage stands extremely significant along with the connectivity.

Coverage is considered extremely significant in the sensor network which aids in assessing and measuring the
QoS (quality of service). There are 3 categories for classifying coverage:

- $\quad$ Area Coverage

- $\quad$ Point Coverage

- Barrier Coverage

Target of area coverage lies in increasing the coverage for ROI (region of interest); set of points are being covered by point coverage and barrier coverage reduces the chances of unidentified invasion via sensor network.

\section{E. Network Reestablishment}

The area needs to be covered by deploying sensor node and the nodes distribution is usually randomly in wide-range environments. The work proposes the deployment of a selforganized node randomly, irrespective of any area. Identical node covering a limited area is taken into consideration. The

nodes have restrictive battery power and working using the residual energy. The nodes life-span remains high if the nodes

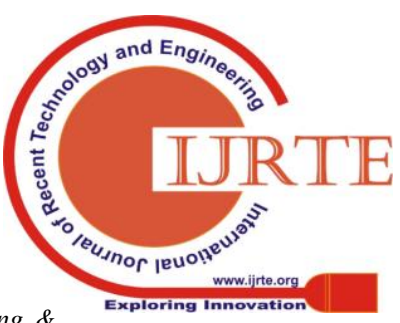

Blue Eyes Intelligence Engineering \& Sciences Publication 


\section{A Robust Method to Detect Coverage Holes and Network Re-Establishment for Nb-Iot Enabled Networks}

successfully preserve their energy while building connection with the neighboring nodes so that the data packets are transmitted till the destination.

\section{F. Store and Forward Technique:}

Communication technique wherein the message that is transmitted from source node which in turn gets stored at an intermediary-device before its being forward towards the destination node. The technique aids in data connectivity and transmission, remote hosts even if there is absence of any direct connection amidst both the nodes(i.e. source and destination). The technique of Store and forward is commonly employed within telecommunication networks, where shortage of dedicated or direct connections is confronted by the remote subscribers. As stated earlier that this technique involves storing of message transmitted and received by the source using an intermediary device, which being a server. The destination node is then identified by the server from the subscribers' database, thereafter the connection is initiated and the stored data packets are then transmitted to the destination.

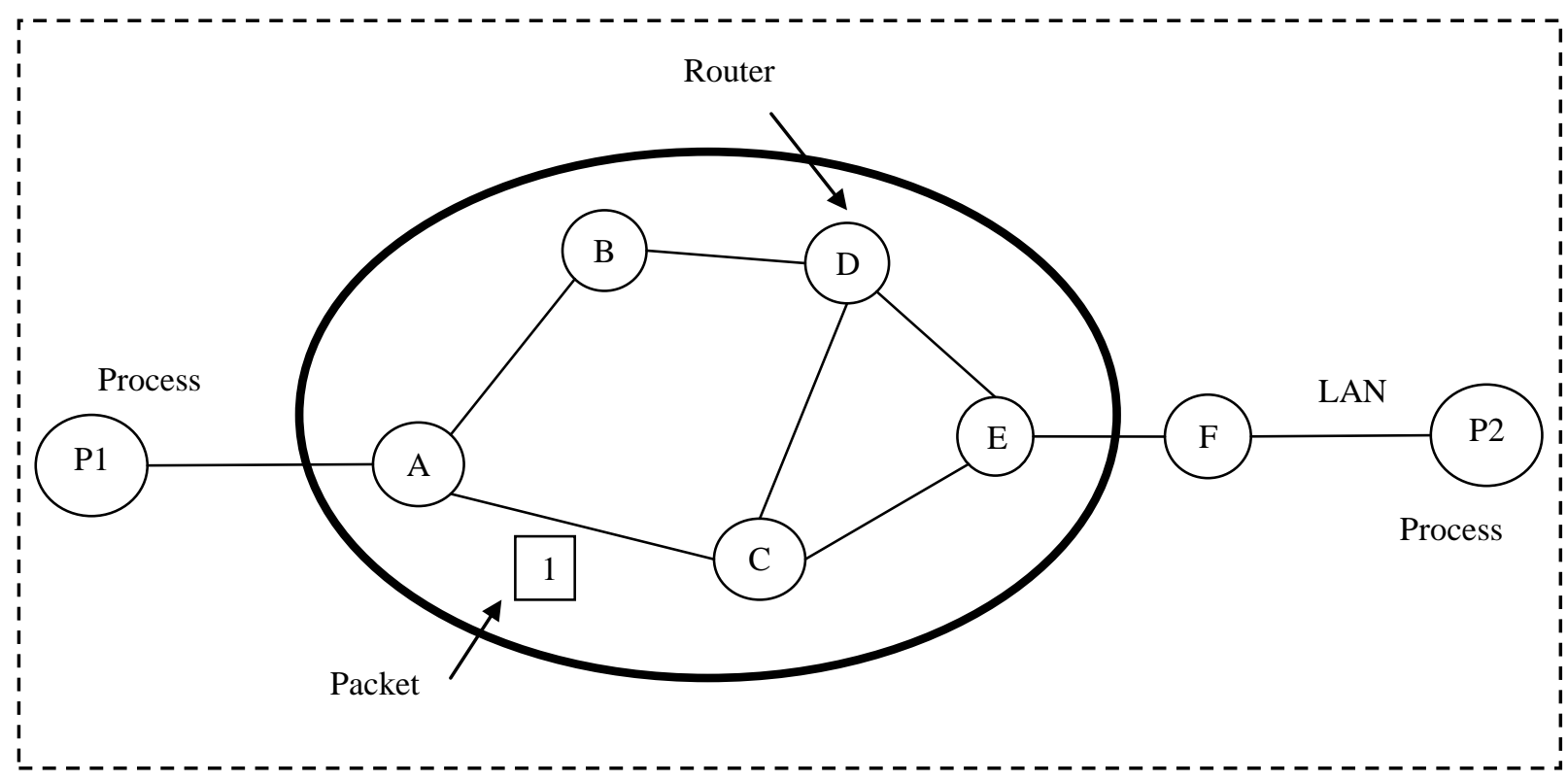

Fig 2: Network layer protocol

\section{G. Algorithm for Store and forward technique}

The network layer functions by routing the data packets from the source end to the destination. The network layer basically looks after selection of routing and the data structures that will be utilized. Network layers routing algorithm helps in determining the output line on which the incoming packet must be transmitted. In case there is use of internal datagram by the subnet, for each incoming packet the decision must be made separately as the most appropriate route might have changed compared to previous time. Subnet that employs virtual circuits, decision are taken once for every session. Routing algorithms are split in 2 main classes: 1 . non adaptive and 2. Adaptive.

\section{Step 1 Start.}

Step 2 identify the neighbors, get the network address.

Step 3 compute the cost or delay for every neighbor.

Step 4 build an effective packet effective with all learnt information.

Step 5 transmit this packet to rest of the routers.

Step 6 Measure the path which is shortest to every single router.
Step 7 identify the coverage hole.

Step 8 determine network stability and re-configuration.

Step 9 Stop.

\section{RESULTS AND DISCUSSION}

Though there is a varied presentation concerning the coverage hole and network re-configuring problems in the prevailing work, there happens to be handful of researches highlighting and emphasizing on coverage holes. Reason for coverage holes being either randomly deployed sensor nodes or energy exhaustion, the challenge lies in identifying these holes, and secondly to repair such holes. The store and forward technique presents few protocols.

Table 1 presents the comparison of Store and forward technique. It depicts the performance of coverage hole and network re-configuring along with the comparison output using various prevailing techniques such as SHORT (Spherical Hole Repair Technique), PreCoRe (Predictive Connectivity Reestablishment), HDRE (Hole Detection

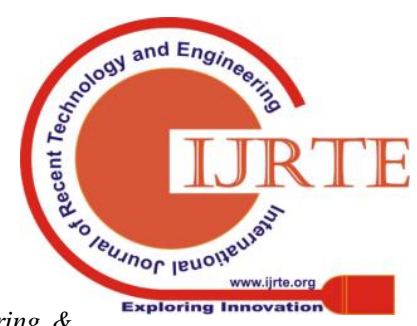

Blue Eyes Intelligence Engineering \& Sciences Publication 


\section{A Robust Method to Detect Coverage Holes and Network Re-Establishment for Nb-Iot Enabled Networks}

Residual Energy). The proposed technique yields in high performance compared to the already prevailing. Thereafter, overall performance such as throughput and time is presented by the work.

Table 1: Comparison Techniques based on coverage hole detection

\begin{tabular}{|l|l|l|l|}
\hline S.No & Techniques & $\begin{array}{l}\text { Throughput } \\
\text { (Mbps) }\end{array}$ & Time(ms) \\
\hline 1 & $\begin{array}{l}\text { Spherical Hole Repair } \\
\text { Technique }\end{array}$ & 1.9 & 0.12 \\
\hline 2 & $\begin{array}{l}\text { Predictive } \\
\text { Connectivity } \quad \text { Re- } \\
\text { establishment }\end{array}$ & 0.35 \\
\hline 3 & $\begin{array}{l}\text { Hole Detection and } \\
\text { Residual Energy }\end{array}$ & 7 & 0.4 \\
\hline 4 & $\begin{array}{l}\text { Store and Forward } \\
\text { Technique }\end{array}$ & 0.10 \\
\hline
\end{tabular}

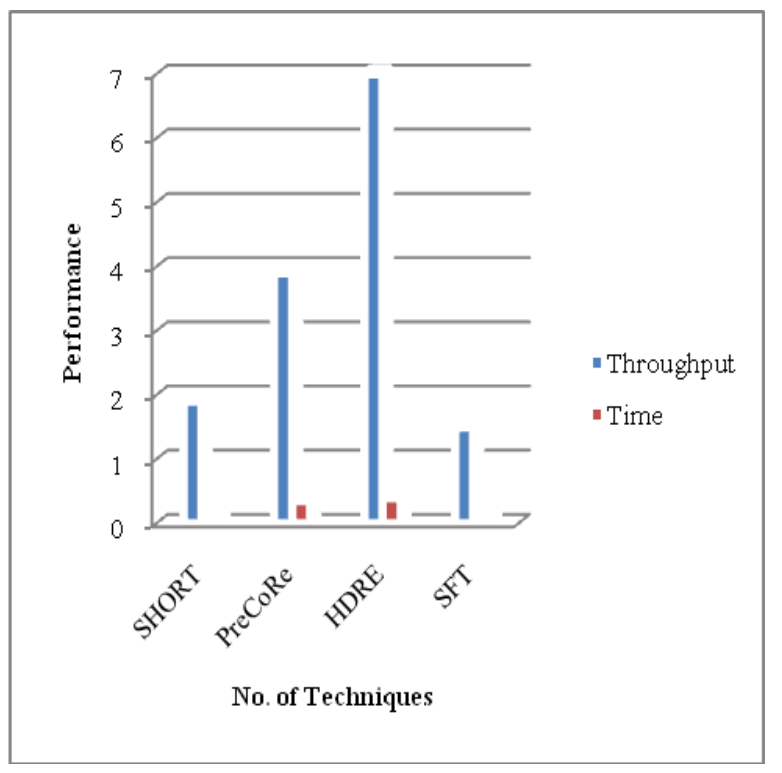

Fig 3: Performance of coverage hole detection techniques

Figure 3 depicts the comparison of store and forward technique. The fig depicts the performance of coverage hole and network re-configuring along with the comparison output using various prevailing techniques such as SHORT (Spherical Hole Repair Technique), PreCoRe (Predictive Connectivity Re-establishment), HDRE (Hole Detection and Residual Energy). The proposed technique yields enhanced output compared to the existing ones.

\section{CONCLUSION}

The WSNs confronts the major issues of Coverage hole and network re- establishment that highly affects the sensor networks quality of service. Various researches worldwide are working on this concerned area by proposing numerable techniques, methodologies and algorithms for resolving this issue. Initially, the research presents an overview about the basic concept of coverage hole. Eventually, based on the networks detecting ability, the locations of coverage holes is being identified with working nodes for fulfilling the energy prerequisite. The proposed method takes into account the impact of working nodes (having short lifespan)in contrast to conventional detection methods. Such implementation of method improvises the practical detection of holes with a reasonable energy being consumed. The proposed method prevents immediate re-occurrence of coverage hole after the network is being reestablished. As a result, effective detection of coverage hole is being offered along with network reestablishment by making use of efficient force based approach.

\section{REFERENCES}

[1] B. Pannetier, J. Dezert, and G. Sella, "Multiple target tracking with wireless sensor network for ground battlefield surveillance," in Proceedings of the 17th International Conference on Information Fusion (FUSION '14), pp. 1-8, IEEE, 2014.

[2] P. K. Sahoo, J.-P. Sheu, and K.-Y. Hsieh, "Target tracking and boundary node selection algorithms of wireless sensor networks for internet services," Information Sciences, vol. 230, pp. 21-38, 2013.

[3] C. Alippi, R. Camplani, C. Galperti, and M. Roveri, "A robust, adaptive, solar-powered WSN framework for aquatic environmental monitoring," IEEE Sensors Journal, vol. 11, no. 1, pp. 45- 55, 2011

[4] J. M. L. P. Caldeira, J. J. P. C. Rodrigues, and P. Lorenz, "Toward ubiquitous mobility solutions for body sensor networks on healthcare," IEEE Communications Magazine, vol. 50, no. 5, pp. $108-115,2012$

[5] Li-Hui Zhao, Wenyi Liu, Haiwei Lei, Ruixia Zhang, and Qiulin Tan "Detecting Boundary Nodes and Coverage Holes in Wireless Sensor Networks",Hindawi Publishing Corporation, Mobile Information Systems, 2016, p.p. 1-16

[6] B. Huang,W.Wu, G. Gao, and T. Zhang, "Recognizing boundaries in wireless sensor networks based on local connectivity information," International Journal of Distributed Sensor Networks,2014, p.p.1-12.

[7] Abdullahi B. Kunya, Gaddafi S. Shehu, Adamu Y. Ilyasu, Sunusi G. Mohammed "Distribution Network Reconfiguration for Loss Reduction and Voltage Profile Improvement using B-PSO", C Research gate, 2016.

[8] Ma, H. C., Sahoo, P. K., \& Chen, Y. W. "Computational geometry based distributed coverage hole detection protocol for the wireless sensor networks", Journal of Network and Computer Applications, 2011, p.p. $1743-1756$.

[9] Ammari, H. M., \& Das, S. "A study of k-coverage and measures of connectivity in 3D wireless sensor networks", IEEE Transactions on Computers, 59(2), 2010, p.p. 243-257.

[10] Babaie, S., \&SajadPirahesh, S. "Hole detection for increasing coverage in wireless sensor network using triangular structure", International Journal of Computer Science Issues, 9(1), 2012, p.p. $213-218$

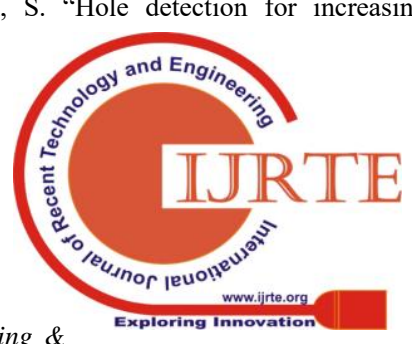




\section{A Robust Method to Detect Coverage Holes and Network Re-Establishment for Nb-Iot Enabled Networks}

[11] Kang, Z., Yu, H., \&Xiong, Q. "Detection and recovery of coverage holes in wireless sensor networks", Journal of Networks, 8(4), 2013 , p.p. $822-828$.

[12] Kamran Latif, Nadeem Javaid, Ashfaq Ahmad, Zahoor Ali Khan, Nabil Alrajeh, Majid Iqbal Khan "On Energy Hole and Coverage Hole Avoidance in Underwater Wireless Sensor Networks", (C) IEEE SENSORS JOURNAL, VOL. 16, NO. 11, 2016, P.P. 4431 4442 .

[13] C. Ma, J. He, H.-H. Chen, and Z. Tang, "Coverage overlapping problems in applications of IEEE 802.15.4 wireless sensor networks," in Proc. IEEE Wireless Commun. Netw. Conf. (WCNC), Apr. 2013, pp. 4364-4369.

[14] P. K. Sahoo and W.-C. Liao, "HORA: A distributed coverage hole repair algorithm for wireless sensor networks," IEEE Trans. Mobile Comput., vol. 14, no. 7, pp. 1397-1410, Jul. 2015.

[15] Yunzhou Zhang, Xiaohua Zhang, Wenyan Fu, Zeyu Wang, and Honglei Liu "HDRE: Coverage Hole Detection with Residual Energy in Wireless Sensor Networks", (C) JOURNAL OF COMMUNICATIONS AND NETWORKS, VOL. 16, NO. 5, 2014, p.p. 1-9.

[16] P. Martins, F. Yan, and L. Decreusefond, "Connectivitybased distributed coverage hole detection in wireless sensor networks," in IEEE Global Telecommunications Conference (GLOBECOM '11), pp. 1-6, 2011.

[17] S. Zeadally, N. Jabeur, and I. M. Khan, "Hop-based approach for holes and boundary detection in wireless sensor networks," IET Wireless Sensor Systems, vol. 2, no. 4, pp. 328-337, 2012.

[18] W.-C. Chu and K.-F. Ssu, "Location free boundary recognition in mobile wireless sensor networks with a distributed approach," Science Direct Computer Network, vol. 70, pp. 96-112, 2014.

[19] Pearl Antil and Amita Malik "Hole Detection for Quantifying Connectivity in Wireless Sensor Networks: A Survey", Hole Detection for Quantifying Connectivity in Wireless Sensor Networks: A Survey, Hindawi Publishing Corporation Journal of Computer Networks and Communications, 2014, p.p. 1-11.

[20] Wei Li, Wei Zhang "Coverage hole and boundary nodes detection in wireless sensor networks", (C) Elsevier Ltd, Journal of Network and Computer Applications, 2014, p.p. 1-9.

[21] Anju Sangwan, Rishi Pal Singh "Coverage Hole Detection and Healing to Enhance Coverage and Connectivity in 3D Spaces for WSNs: A Mathematical Analysis", Springer, Wireless PersCommun, 2017.

\section{AUTHORS PROFILE}

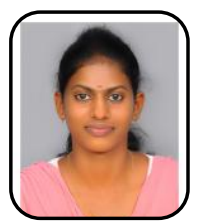

Anbumani M received the B.E, degree in Computer Science and Engineering from Jeppiaar Institute of Technology and pursuing Master of Engineering in Department of Computer Science and Engineering from SRM Institute of Science and Technology. Anna University, Chennai in 2016. She is currently an Assistant Professor in SRM Institute of Science and Technology (formerly known as SRM University), Her Research interests in Natural Language Processing, Information Retrieval, Information Abstraction and Ontology.

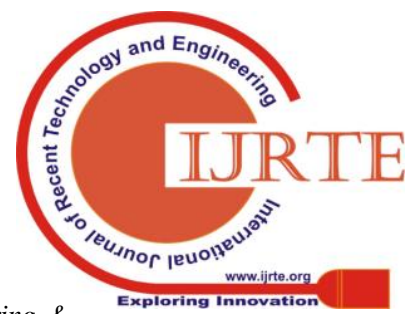

